\title{
Research into Synthesizing Access Control Strategies in Cloud Computing Environment
}

\author{
Ping Ni \\ The library of Hubei institute for nationalities, Enshi, Hubei 445000, China \\ nnpping@163.com
}

Keywords: Cloud computing environment; Access control; Strategy synthesis

\begin{abstract}
The development of computer technologies and the Internet technology has promoted the upgrading of cloud computing, and application mode of information technology also begins to be transferred from the traditional centralized mode to the distributed morphology gradually while people are constantly having higher requirements for information sharing. The effective application of cloud computing can satisfy people's actual demands in daily work. However, in order to use cloud computing to realize the cross-border collaboration, perfect orientation control strategy should be developed as the guarantee. This paper conducts the research from three aspects: cloud computing, access control and strategies, hoping to promote better development of cloud computing.
\end{abstract}

\section{Introduction}

In recent years, cloud computing has been developing rapidly, and all aspects of it have been becoming perfect and sound, so cloud computing is gradually receiving the attention and concern from various industries. Cloud computing stores the vast amount of dispersed data onto the "cloud" so as to lay foundation for better information sharing as well as provide users with rapid, targeted and dynamic IT services accordingly. In terms of the actual situation at present, cloud computing still needs to face various problems in the improvement, and the most crucial one is the security of cloud computing. It is not difficult to find from researches into the security of cloud computing that the quality of access control will directly determine the security and completeness of cloud computing data. Therefore, it has become very important to enhance researches into the access control strategies.

\section{Brief of Cloud Computing}

\section{Brief of Cloud Computing Technology.}

Concept.Cloud computing is a brand new form of network service that has been proposed and develops in recent years. With the Internet as the core foundation, cloud computing puts traditional form of computer services onto the cloud so as to achieve effective transfer between the client and the network. Therefore, the importance of network in cloud computing cannot be ignored, and various resources and data stored in the cloud should be transferred to users through the Internet, so once the Internet cannot work, the transfer cannot be conducted normally [1].

Cloud computing is established on grid computing, parallel computing and distributed process, and under such background, previous traditional data and resources will be stored in the cloud server so that users can make full and effective use of these computer resources. With the constant development and accomplishment of various aspects of cloud computing, cloud computing has already become a main trend in the development of computer technology and it also meets the current requirement of being low-carbon and green. Also, with good advantages, cloud computing has already attracted various enterprises and government sectors to study and establish it because they hope to occupy the dominant position in the cloud computing market.

Service Modes. At present, there are three classic service models of cloud computing: IaaS, PaaS, and SaaS, as shown in Table 1. In the following, these three service modes are analyzed respectively. 
Firstly, IaaS is the abbreviation of Infrastructure as a Service. In this service mode, the computing ability and storage ability are used by users as commodities while users need to utilize their own computer resources according to corresponding charging standards and ways. Generally, this services regards the Internet as its corresponding service carrier and the IT implementation ability is provided to corresponding users through the virtual server. In terms of the usage of the IaaS service mode, it has a lot of characteristics. For example, computing can be conducted according to users' actual usage of resources; the Internet can be used as the transfer media and tool; and usage of resources and data can be effectively supervised, controlled and managed.

Secondly, PaaS is the abbreviation of Platform as a Service. When relevant enterprises develop IT, they can use the PaaS to obtain the resources and environment they need so as to transfer the development contents onto this platform. This service mode has many advantages. For example, it has various kinds of services, it can be expanded with a high level of integrating resources, and the development environment is very safe, etc. [2].

Finally, SaaS is the abbreviation of Software as a Service. In this mode, users no long need to purchase corresponding maintenance software as before and they can obtain corresponding service by paying some fees to the software developers. Software developers can provide services to users through the Internet. However, it should also be noted that this service mode does not have good configuration ability and there is certain limit to itself.

Table 1 One Platform Service Modes

\begin{tabular}{|l|l|}
\hline Service Mode & Cloud Computing Platform \\
\hline SaaS & SalesForce, Google Docs \\
\hline PaaS & $\begin{array}{l}\text { Amazon Elastic Map } \\
\text { Reduce、 } \\
\text { Microsoft Azure Service 、 } \\
\text { Google App Engine }\end{array}$ \\
\hline IaaS & $\begin{array}{l}\text { Amazon EC 2 G GoGid, } \\
\text { Amazon S3 }\end{array}$ \\
\hline
\end{tabular}

Cloud Computing Framework. With the rapid development of the Internet, the actual application range of cloud computing service platform is gradually expanding, and it is effectively transferring a huge amount of computing and storage capacity onto this platform gradually so as to provide users with more rapid services. After the virtual mode is used, computing ability of the independent server is integrated into a corresponding whole, i.e. the cloud computing platform. Diagram of the cloud computing system's structure is as shown in the following Fig. 1.

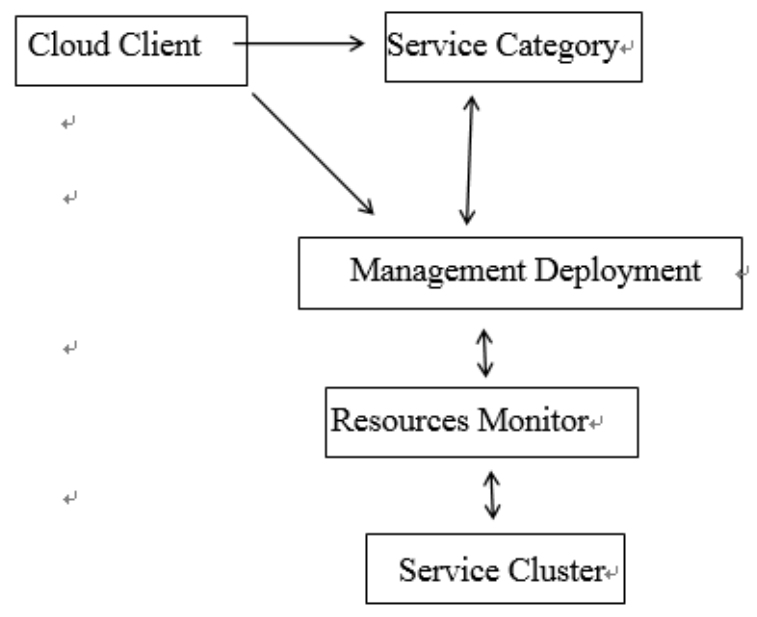

Figure 1. Diagram of the Cloud Computing System's Structure 


\section{Key Technologies}

Virtual Technology. In cloud computing, virtual technology (as shown in the following Fig. 2) is an indispensable technology in the development and construction process. The fundamental purpose of this technology is to scientifically, reasonably and dynamically distribute the computing resources while virtual technology can operate various software or operation systems effectively in the same hardware system so as to make full use of the hardware resources. During the virtualization process, corresponding construction equipment is put in the middle of the hardware resources and operation system with certain functions. Virtual technologies can not only effectively manage and control hardware resources, but also guarantee the hardware resources are effectively utilized. Also, it can effectively transfer the hardware resources to the last layer of operation system to guarantee different operation systems can be operated independently without interfering with each other [3].

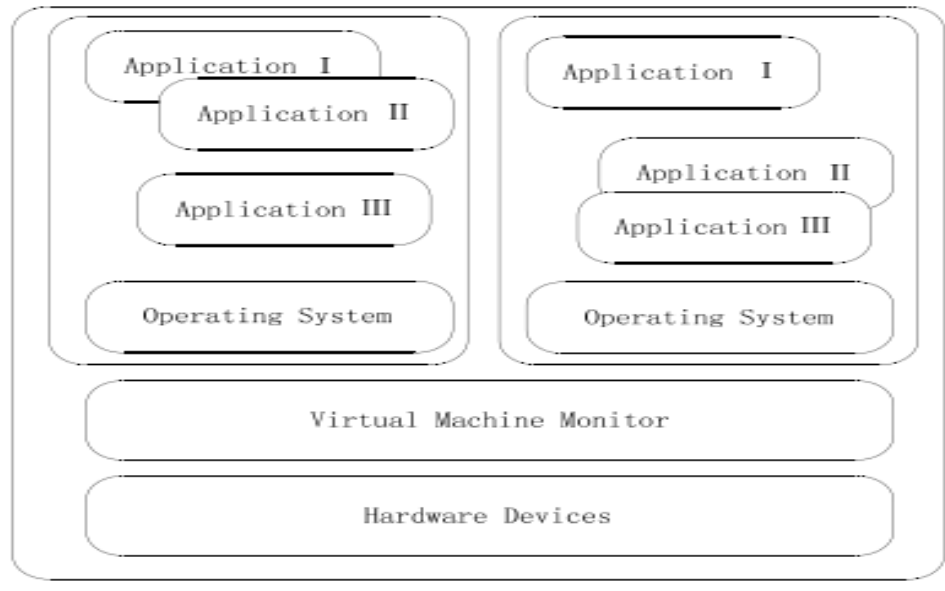

Figure 2. Virtualization Figure

Storage Technology. Cloud computing platforms have a lot of data processing, so you want a complete content you need to use a distributed system as the support of its data processing, while the distributed storage can be at the same time for many users of data for effective treatment, and it is also good to adapt cloud computing environments. So this also requires users to use a distributed system, not to the more specific details of which promoted understanding, to make full use of the API interface can be achieved on the file upload, modify, and delete operations. [4] Accordingly distributed storage in the cloud, not just stored on the server, but the data is stored to the appropriate cluster. on the next phase of the actual situation, the more mainstream cloud computing the system will be able to achieve the purpose of the massive data store count and processed.

Parallel Programming Technology. Because cloud computing will be a lot of data processing, so to give the corresponding open big personnel has brought great pressure, so in the actual development process should be simplified programming model, so as to achieve the purpose of processing large data now to stage the most widely used programming model is developed by the company have Google's MapReduce to the data parallel computing, and data collection is usually more than 1TB its practical application process can be divided into two steps, first, to the corresponding data mapping, making it map. Second, then the data generated to regulate and simplify. and in practical application process where users do not have to pay attention to details, so that it can effectively improve their data processing effectiveness.

Security of Cloud Computing. It can be known from developing and applying cloud computing that during the development of cloud computing, the several following aspects should be considered:

First of all, connection of privileged users. As the storage mode has been transferred, more and more enterprises ask servers to store high confidential documents and such sensitive data storage definitely has potential risks.

Secondly, the issue of supervision. Although enterprises store important data at servers, they should still be responsible for the security and completeness of these data. Therefore, cloud 
computing servers have to ask a third party to inspect or manage the security of data seriously so as not to threaten data. Otherwise, it will definitely bring serious risks and threat.

Thirdly is the issue of data's location. Most users have already uploaded data onto the cloud computing platform, but they do not know the storage location. There is also such problem with enterprise users, so distributed storage of cloud computing will cause problems in supervision and both individual users and enterprise users need to get to know where data are exactly stored before choosing cloud services.

The fourth is data isolation. Because cloud computing platform will be used to store data in the form of distributed, but in the context of a network share, the data itself will also face many problems, although the encryption mode to solve some of these problems, but not all problems can be eradicated, but data encryption will be in some way hinder the use of data encryption level, so data security is necessary to reduce the isolation suffered by the way.

The fifth is data recovery. If data are lost during the storage process during to service failure, it will definitely cause serious loss to users, so it is necessary to copy and manage data in copy backup so that even if there is failure, data can be recovered [5].

The sixth is to investigate support. If users want to investigate cloud services and collect data, they will face serious restriction because many records in cloud involve numerous users, so the investigation process involves various aspects and such behavior will not get support.

The last is the issue about survival. Although in ideal state, cloud computing servers will continue to operate, it cannot rule out cases of merging or bankruptcy. Therefore, once such case occurs, it will cause serious threat to the stability of users' data.

\section{Cloud Computing Access Control}

The cloud computing system is composed of three parts: basic services, application services and infrastructure services in order to guarantee the security of cloud services and effectively protect users' security and privacy.

In the context of the information environment, access control is to ensure that appropriate information will not be available illegally in its excellent safety systems, after appropriate authorization objects to the requested resource for data access, whereas the corresponding object is will not qualify for the requested resource. In simple terms, the fundamental purpose of the visit is to get control whether resources legality verification and validation, access control and therefore is also an act of authorization, the effective use of the access control system capable of running their own security evaluation and OK. Control access to the three elements and access policy rules, visit the main and access objects. When the data resource protection, access control will be considered as the game referee, but the body would like to get their own access the required information, we need to access the object, but only authorized access control its operation and certainly after, can the object be to continue to visit, and this will put an end to the information to be malicious removal, theft or tampering. The subject and object of Inter has always maintained a dynamic structural changes, meaning that once the body is accessed, it can also become appropriate. against increasing the access control application access, using its cross-domain access is rising, while the traditional control model we cannot meet the actual needs of the development of cloud computing, and therefore be able to meet the need to explore the background of cloud computing access control policy, to further ensure the security and stability of the data.

\section{Effective Ways to Synthesize the Access Control Strategies in Cloud Computing Background}

Control Mode Constructed based on Cloud Computing's Attributes. Development of cloud computing makes distributed systems develop towards huge, complex direction of change, therefore, it will cause the control system requirements for information security have a higher level, coupled with cloud computing to shift resources to the cloud so that different types of more complex link between the cloud and cumbersome, the traditional access control model cannot meet the actual 
demand, coupled with the classical control mode cannot effectively accommodate multi-domain environment at this stage and to some extent also hinder efficiency as well as to enhance the stability, and the cloud computing environment requires fine-grained access control, so that the traditional model of the past cannot meet its requirements. Therefore, access control model is established based on the property has been effective development, which not only can be used as the final decision basis, but also to better adapt to meet the actual change properties, and then targeted to provide an update strategy.

\section{Strategy Synthesis Scheme}

Basic Ideology. Synthesis of access control policy must be in accordance with the requirements of cloud computing environments synthesis proposed to carry out, its strategy is to expand the traditional model of the body, resources, the environment and the corresponding authorization, etc., making it subject, resource, operating, the environment and safety, while operating elements extend beyond the equivalent authority elements, and this synthetic scheme security element itself also has a corresponding attribute range, able to apply different register access confidential registration under different environments, so to the role of security attributes, it is possible to effectively distinguish and refine control strategies so that their own fine-grained policy effectively improved, therefore, expand its elements is essential. access control policy synthesis process is as follows Fig. 3 shows.

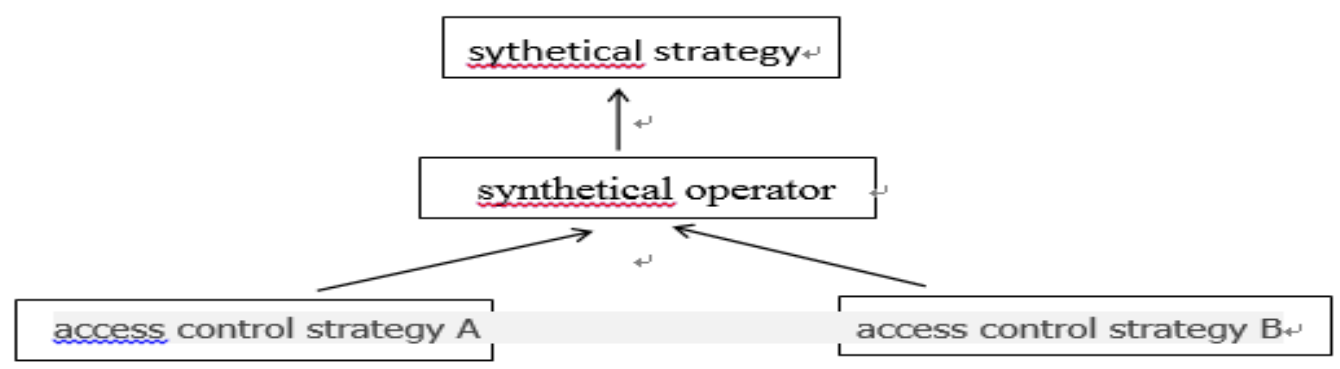

Figure 3. Diagram of Strategy Synthesis

In this scheme, the databases A and B have developed a corresponding access policies, its property authorized entry binding, it is the body of information resources, operations, operating environment, and access security of the composition. If the access subject to when the appropriate cross-domain information access, synthesize operator will be on A and B are synthesized access policy, and then combined into a new strategy to achieve the purpose of access control. the database in the cloud computing environment than in the past tradition database has more dynamic, so that the environment and the situation becomes more complex and dynamic access control. and the root cause of this phenomenon is because the access control environment is not the same body, so it also gives control strategy synthesis brought certain challenges.

Analysis of Synthesis Methods. In the previous centralized environment, integrated semantic synthesis strategies can be relatively simple synthetic structure operator to resolve, although to some extent, and role mapping static environment can meet the needs of policy synthesis, however, multi-domain and dynamic policy synthesis requirement not be satisfied, therefore, we need to build to meet the policy synthesis cloud computing environments. the strategy based on the development of synthetic property not only has a good performance to adapt to heterogeneous environments, the ability to resolve policy conflicts, but also has very good semantic skills, these advantages and characteristics are able to adapt and meet the distributed environment of their own characteristics and requirements.

\section{Conclusion}

In short, with the rapid development of cloud computing technology, there are more and more application established based on it and most enterprises and individuals begin to direct store data on 
the cloud. Therefore, the development of cloud computing has also brought new challenge to itself, and access control security in the cloud as an indispensable part of the relevant developer must attach importance to them, and in-depth study, but at this stage there is a corresponding strategy of its limitations in access control, so we need to have a good synthetic strategy to protect its control, thereby enabling access control security level is improved.

\section{References}

[1] Shangguang Wang,Zhipiao Liu,Qibo Sun et al.Towards an accurate evaluation of quality of cloud service in service-oriented cloud computing[J].Journal of Intelligent Manufacturing,2014,25(2):283-291.

[2] Muhammad Irfan,Zhu Hong,Tauseef Qamer et al.Requirement Analysis and Design of Service Level Integration Layer for Cloud Computing Services, to Meet Service Level Agreements and Quality of Service[J].Journal of computational and theoretical nanoscience,2014,11(3):629-636.

[3] Abdul Nasir Khan,M. L. Mat Kiah,Sajjad A. Madani et al.Incremental proxy re-encryption scheme for mobile cloud computing environment[J].Journal of supercomputing,2014,68(2):624-651.

[4] Md Whaiduzzaman,Mehdi Sookhak,Abdullah Gani et al.A survey on vehicular cloud computing[J].Journal of network and computer applications,2014,40:325-344.

[5] Thamarai Selvi Somasundaram,Kannan Govindarajan.CLOUDRB: A framework for scheduling and managing High-Performance Computing (HPC) applications in science cloud[J].Future generations computer systems: FGCS,2014,34:47-65. 